\title{
Control of PD-L1 expression by miR-140/142/340/383 and oncogenic activation of the OCT4-miR-18a pathway in cervical cancer
}

\author{
Peixin Dong ${ }^{1,2} \cdot$ Ying Xiong ${ }^{3} \cdot$ Jiehai $\mathrm{Yu}^{3} \cdot$ Lin $\mathrm{Chen}^{3} \cdot$ Tang Tao $^{4} \cdot$ Song $\mathrm{Yi}^{4} \cdot$ Sharon J. B. Hanley ${ }^{1}$ Junming Yue, ${ }^{5,6}$ \\ Hidemichi Watari ${ }^{2} \cdot$ Noriaki Sakuragi $^{1}$
}

Received: 19 November 2017 / Revised: 20 March 2018 / Accepted: 1 May 2018

(c) The Author(s) 2018. This article is published with open access

\begin{abstract}
PD-L1, a key inhibitory immune receptor, has crucial functions in cancer immune evasion, but whether PD-L1 promotes the malignant properties of cervical cancer (CC) cells and the mechanism by which PD-L1 is regulated in CC remains unclear. We report that PD-L1 is overexpressed in CC, and shRNA-mediated PD-L1 depletion suppresses the proliferation, invasion, and tumorigenesis of CC cells. Loss of miR-140/142/340/383 contributes to PD-L1 upregulation. miR-18a enhances PD-L1 levels by targeting PTEN, WNK2 (ERK1/2 pathway inhibitor), and SOX6 (Wnt/ $\beta$-catenin pathway inhibitor and p53 pathway activator) to activate the PI3K/AKT, MEK/ERK, and Wnt/ $\beta$-catenin pathways and inhibit the p53 pathway, and miR-18a also directly suppresses the expression of the tumor suppressors BTG3 and RBSP3 (CTDSPL). miR-18a overexpression in $\mathrm{CC}$ cells is triggered by OCT4 overexpression. Our data implicate PD-L1 as a novel oncoprotein and indicate that miR-140/ 142/340/383 and miR-18a are key upstream regulators of PD-L1 and potential targets for CC treatment.
\end{abstract}

These authors contributed equally: Peixin Dong, Ying Xiong.

Electronic supplementary material The online version of this article (https://doi.org/10.1038/s41388-018-0347-4) contains supplementary material, which is available to authorized users.

Peixin Dong

dpx1cn@gmail.com

$\triangle$ Junming Yue jyue@uthsc.edu

$\triangle$ Hidemichi Watari

watarih@med.hokudai.ac.jp

1 Department of Women's Health Educational System, Hokkaido University School of Medicine, Hokkaido University, Sapporo 0608638, Japan

2 Department of Obstetrics and Gynecology, Hokkaido University School of Medicine, Hokkaido University, Sapporo 0608638, Japan

3 Department of Gynecology, State Key Laboratory of Oncology in South China, Sun Yat-Sen University Cancer Center, 510060 Guangzhou, China

4 Faculty of Medicine, Department of Obstetrics \& Gynaecology, The Chinese University of Hong Kong, Shatin, New Territories, Hong Kong, China

5 Department of Pathology and Laboratory Medicine, University of Tennessee Health Science Center, Memphis, TN 38163, USA

6 Center for Cancer Research, University of Tennessee Health Science Center, Memphis, TN 38163, USA

\section{Introduction}

Cervical cancer (CC) is the fourth most common malignancy in women and the fourth leading cause of cancerrelated deaths among women worldwide [1, 2]. Tumors suppress the host immune system by upregulating programmed death ligand 1 (PD-L1) that binds to programmed death-1 on $\mathrm{T}$ cells, resulting in inhibitory checkpoint signaling that inhibits $\mathrm{T}$ cell expansion and function [3-5]. Overexpression of PD-L1 has been found in human cancers, including $\mathrm{CC}$ and pancreatic cancer [6-8]. In addition to mediating $\mathrm{T}$ cell suppression, recent studies have shown the critical roles of PD-L1 in promoting cancer cell growth and invasion [9-11]. However, the exact biological function of PD-L1 in CC remains unclear.

EGFR mutation, PTEN deletion, PI3K or AKT mutations, aberrant JAK/STAT signaling, and Wnt/ $\beta$-catenin signaling activation can induce PD-L1 expression [12-16]. MicroRNAs (miRNAs) are critical regulators of cancer metastasis [17-19]. miR-513 and miR-570 target PD-L1, while p53 indirectly inhibits PD-L1 levels by inducing miR34a expression [20-22]. The miRNAs that have the capacity to modulate PD-L1 expression in CC remains unknown.

We hypothesize that PD-L1 not only promotes tumor immune escape, it also enhances the malignant properties of $\mathrm{CC}$ cells. In the present study, we found that PD-L1 is 

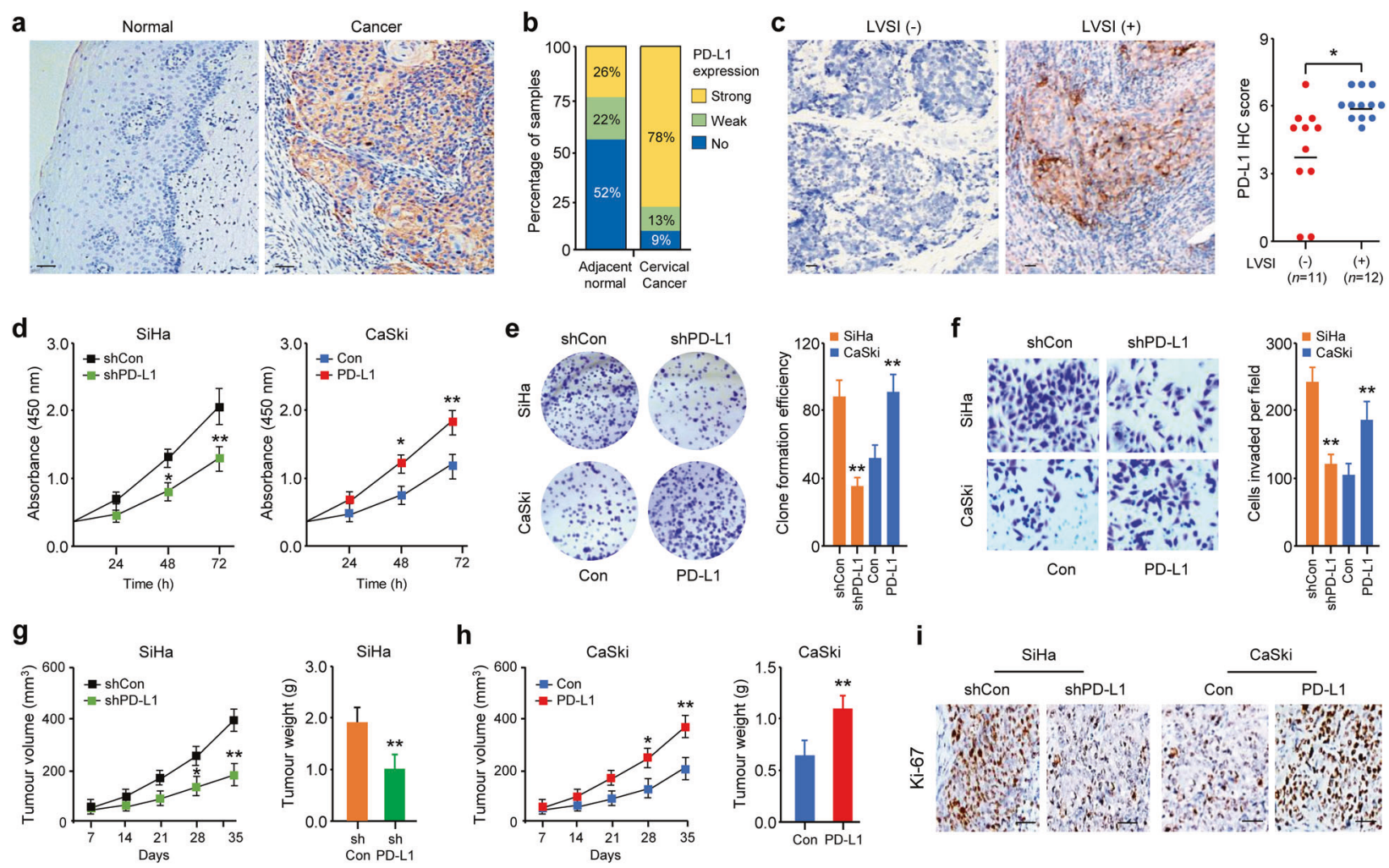

i
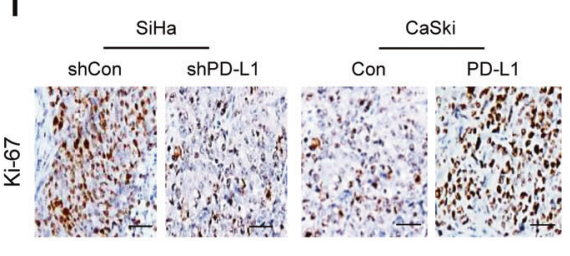

Fig. 1 PD-L1 is upregulated in cervical cancer patients and is required for tumor growth and invasion. a Representative immunohistochemistry (IHC) image of PD-L1 in 23 paired human cervical cancer (CC) and adjacent normal cervical specimens (scale bar: $50 \mu \mathrm{m}$ ). b Percentage of cases that exhibit different PD-L1 protein expression levels (no staining, weak, or strong) in 23 paired human $\mathrm{CC}$ tissues and adjacent normal tissues $(P<0.01)$. $\mathbf{c}$ Representative IHC images of PD-L1 (left panel) and scatter plots showing the IHC scores for PD-L1 protein in patients with or without lymphovascular space invasion (LVSI, right panel). Scale bar: $50 \mu \mathrm{m}$. d Growth curves of PD-L1knockdown SiHa cells and CaSki cells with ectopic PD-L1 expression

overexpressed in $\mathrm{CC}$ and is an important promoter of $\mathrm{CC}$ cell proliferation and invasion. We also identify two novel mechanisms, including a miR-140/142/340/383-PD-L1 axis and an OCT4-miR-18a-PTEN/WNK2/SOX6 axis, that are responsible for the upregulation of oncoprotein PD-L1 in CC, suggesting that targeting PD-L1 by introducing miR140/miR-142/miR-340/miR-383 or silencing of miR-18a might represent a therapeutic option to repress the metastatic phenotypes of CC cells and simultaneously reverse the immunosuppressive $\mathrm{CC}$ microenvironment.

\section{Results}

\section{PD-L1 is aberrantly expressed in primary CC samples and CC cell lines}

We evaluated PD-L1 expression using immunohistochemical (IHC) analysis of 23 primary CC and paired adjacent

$(n=6)$. e Representative images (left panel) and data quantification (right panel) of colony formation of PD-L1-knockdown SiHa cells and CaSki cells with ectopic PD-L1 expression $(n=6)$. f Representative images (left panel) and data quantification (right panel) of Matrigel invasion assays of PD-L1-knockdown SiHa cells and CaSki cells with ectopic PD-L1 expression $(n=6)$. $\mathbf{g}, \mathbf{h}$ Growth curves and quantification of weight of tumors that developed in nude mice injected subcutaneously with PD-L1-knockdown SiHa cells (g) or PD-L1overexpressing CaSki cells (h) $(n=8$ per group). i Representative IHC images of Ki-67 in tumors from the mice described in $\mathbf{g}, \mathbf{h}$. Scale bar: $50 \mu \mathrm{m} . * P<0.05, * * P<0.01$

normal tissue specimens. A strong PD-L1 staining was observed in CC samples (Fig. 1a). 78\% of the cancer tissues displayed strong PD-L1 expression, whereas most adjacent normal samples (74\%) showed no or weak PD-L1 expression $(P<0.01$, Fig. $1 b)$. The staining intensity of PD-L1 in $\mathrm{CC}$ was significantly higher than that in adjacent normal samples $(2.61 \pm 0.19$ vs. $1.0 \pm 0.14, P<0.01)$. Higher PDL1 expression levels were associated with a higher incidence of lymphovascular space invasion (Fig. 1c).

We next assessed PD-L1 expression in a panel of CC cell lines and the immortalized human endometrial epithelial EM cell line using immunoblotting. Compared with EM cells that do not have significant PD-L1 expression, CC cell lines overexpressed PD-L1 (Supplementary Fig. 1a). The highest level of PD-L1 was observed in SiHa cells, which have a high capacity to invade in a Matrigel cell invasion assays [23] (Supplementary Fig. 1a), suggesting that PD-L1 may function as an oncoprotein in $\mathrm{CC}$. 


\section{PD-L1 promotes CC cell proliferation and invasion}

To examine the contribution of endogenous PD-L1 to the proliferative and invasive phenotype of $\mathrm{CC}$ cells, we performed PD-L1 loss-of-function and gain-of-function experiments in CC cell lines (Supplementary Fig. S1a). Short hairpin RNA-mediated knockdown of PD-L1 decreased $\mathrm{SiHa}$ cell proliferation and invasion, and stable overexpression of PD-L1 in CaSki cells promoted their proliferation and invasion (Fig. 1d-f).

We next explored the biological function of PD-L1 in CC tumorigenesis in vivo by injecting PD-L1-knockdown SiHa cells or PD-L1-overexpressing CaSki cells into nude mice. PDL1-knockdown SiHa cells generated smaller tumors, and PDL1-overexpressing CaSki cells formed larger tumors than control cells (Fig. 1g, h). PD-L1-depleted SiHa tumors exhibited downregulation of Ki-67, but PD-L1-overexpressing CaSki tumors showed upregulation of Ki-67 (Fig. 1i). To examine whether the cytoplasmic domain of PD-L1 plays a critical role in promoting CC cell invasion and proliferation, we overexpressed either wild-type (WT) PD-L1 or cytoplasmic domain-truncated PD-L1 and observed that CaSki cells overexpressing WT PD-L1, but not cytoplasmic domain-truncated PD-L1, exhibited significantly higher proliferation and invasion than the control cells (Supplementary Fig. S1a), suggesting that PD-L1 induces CC cell proliferation and invasion.

\section{Identification of miRNAs that regulate PD-L1 expression}

To identify miRNAs that suppress $\mathrm{CC}$ invasion and metastasis, we isolated highly invasive subpopulations of $\mathrm{SiHa}$ cells using Matrigel Invasion Chambers [24]. In contrast to parental SiHa cells, the majority of invasive $\mathrm{SiHa}$ cells exhibited a spindle-shaped and mesenchymallike morphology (Supplementary Fig. S2a). Compared with the parental cells, the invasive $\mathrm{SiHa}$ sublines showed enhanced invasion abilities (Supplementary Fig. S2a).

Then, a miRNA microarray assay was performed, and the expression profiles of invasive $\mathrm{SiHa}$ cells and their parental cells were determined (Fig. S2b; Supplementary Table S1). We merged the obtained list of downregulated miRNAs in highly invasive SiHa cells obtained by microarray assay with miRNAs predicted by an in silico analysis, and identified miR-140/142/340/383 as potential regulators of PD-L1 in CC cells (Supplementary Fig. S1c). Similarly, we defined miR-18a, which was upregulated in highly invasive SiHa cells (Supplementary Figs. S1c, S2b), and may act as the suppressor of PTEN, an upstream repressor of PD-L1 [3].

Real-time RT-PCR (qRT-PCR) assay confirmed that miR-140/142/340/383 were significantly downregulated, whereas miR-18a was significantly overexpressed in CC cell lines compared with normal EM cells (Supplementary Fig. S1d). qRT-PCR assay validated the lower miR-140/ $142 / 340 / 383$ expression or higher miR-18a expression in 60 primary $\mathrm{CC}$ tissues relative to matched adjacent normal tissues (Supplementary Fig. S2c). In CC samples, $P D-L 1$ expression was positively correlated with miR-18a expression, but inversely correlated with miR-140/142/340/383 expression (Supplementary Fig. S2d). CC patients with higher miR-18a expression or lower miR-140/142/340/383 expression had a shorter survival time (Supplementary Fig. $\mathrm{S} 2 \mathrm{e})$.

We tested whether $P D-L 1$ mRNA expression is regulated by these identified miRNAs. Transient transfection of the miR-140/142/340/383 mimic or anti-miR-18a inhibitor reduced PD-L1 expression in SiHa cells. Conversely, transfection of the miR-18a mimic or anti-miR-140/142/ 340/383 inhibitors increased PD-L1 expression in CaSki cells (Supplementary Fig. S1e, f).

\section{PD-L1 is directly repressed by the miR-140/142/340/ 383 tumor suppressors}

We performed the luciferase reporter assays by cotransfecting CC cells with a luciferase reporter plasmid fused to WT PD-L1 3'-UTR or mutant PD-L1 3'-UTR harboring mutations in the putative miR-140/142/340/383 binding sites, together with miR-140/142/340/383 mimics or anti-miR-140/142/340/383 inhibitors. The luciferase activity of the WT $P D-L 1$ reporter was reduced by miR140/142/340/383 overexpression, but induced by anti-miR140/142/340/383 inhibitors in CC cells (Fig. 2a-c). Mutation of the binding sites abolished the effects of miR-140/ $142 / 340 / 383$ on the luciferase activity (Fig. 2a-c). miR-140/ 142/340/383 overexpression decreased PD-L1 protein expression, and knockdown of these miRNAs increased the PD-L1 protein levels in CC cells (Fig. 2d), indicating that miR-140/142/340/383 directly target the PD-L1 3'-UTR.

Ectopic PD-L1 expression in SiHa cells overcame the suppressive effects of miR-140/142/340/383 mimics on cell proliferation and invasion (Supplementary Fig. S3). SiRNAmediated PD-L1 silencing in CaSki cells suppressed the proliferation and invasion, which were promoted by antimiR-140/142/340/383 inhibitors (Supplementary Fig. S3), suggesting that the PD-L1 mediates the suppressive effects of miR-140/142/340/383.

\section{miR-18a promotes CC cell proliferation and invasion}

We investigated the biological role of miR-18a in CC cells, and found that downregulating miR-18a levels in $\mathrm{SiHa}$ cells decreased proliferation and invasion, whereas overexpressing miR-18a in ME-180 and CaSki cells increased proliferation and invasion (Supplementary Fig. S4a, b). To 
a
Position 848-855 of PD-L1 3' UTR
hsa-miR-140-5p

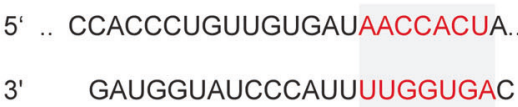
Position 2498-2504 of $5^{4}$.. UUGGAUUUGUAAGGCACUUUAU. PD-L1 3' UTR
hsa-miR-142-5p
$3^{\prime}$
UCAUCACGAAAGAUGAAAUAC
Position 950-956 of
PD-L1 3' UTR
hsa-miR-340-5p
5‘.. GCCACCCACUGUCCUUUUAUAAU..
3' UUAGUCAGAGUAACGAAAUAUU
Position 16-22 of PD-L1 3' UTR
hsa-miR-383-5p
5‘.. UCCAGCAUUgGaAcuUCugaucu.
3' UCGGUGUUAGUGGAAGACUAGA

d
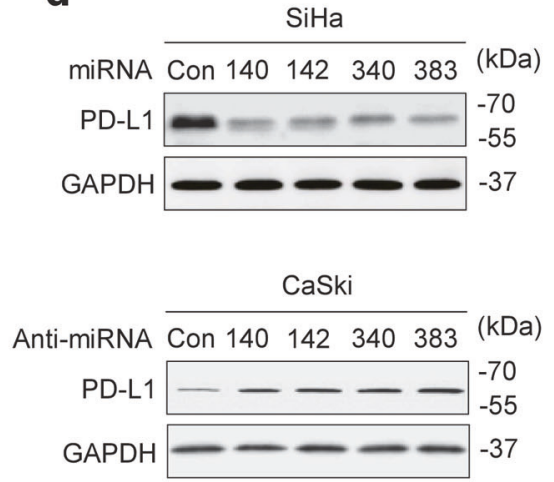

b

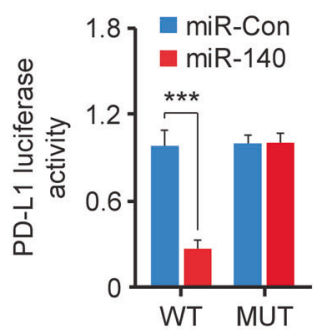

C

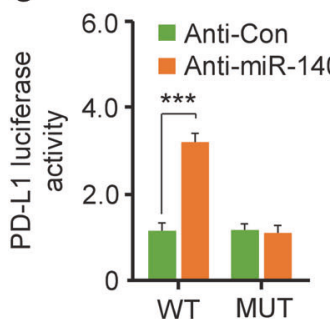

$\mathrm{SiHa}$
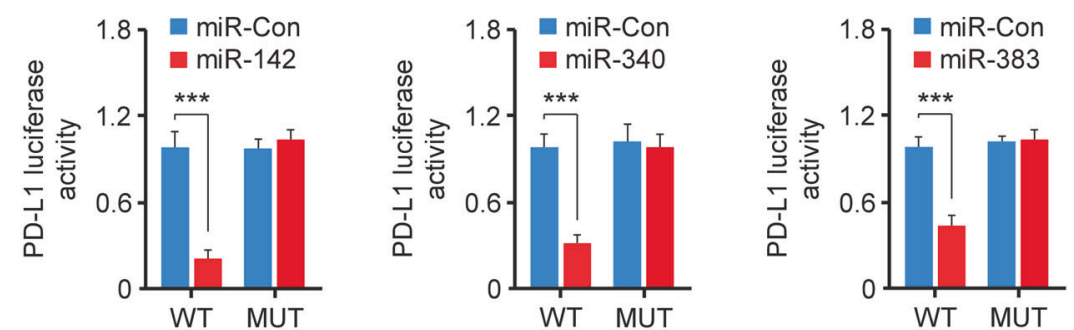

CaSki
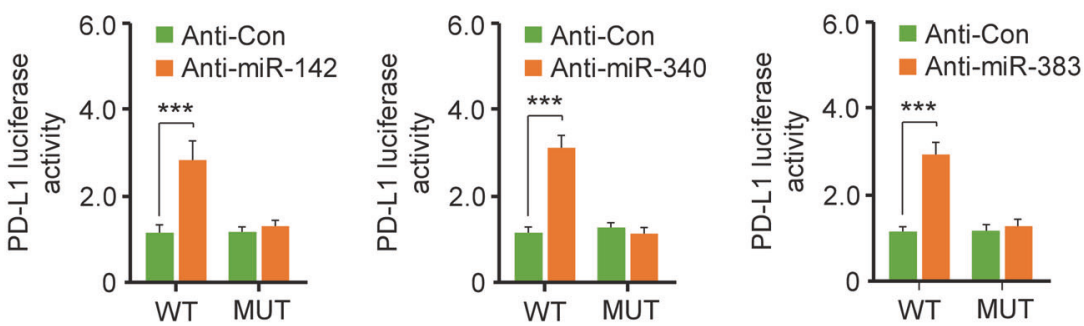

Fig. 2 PD-L1 is directly repressed by the miR-140/142/340/383 tumor suppressors. a Predicted miR-140, miR-142, miR-340, and miR-383 binding sites in the $3^{\prime}$-UTR of $P D-L 1$. b, $\mathbf{c}$ Luciferase reporter assays of SiHa cells co-transfected with a reporter construct containing the wild-type (WT) or mutant (MU) 3'-UTR of PD-L1 and the indicated miRNA mimics or their controls (b) and of CaSki cells co-transfected with a reporter construct containing the WT or MU 3'-UTR of PD-L1 and the indicated anti-miRNA inhibitors or their negative controls $(\mathbf{c})(n=4)$. d Western blot analysis of PD-L1 in SiHa (upper panel) and CaSki (lower panel) cells transfected as indicated $(n=3)$. $* * * P<0.001$

further explore the cellular function of miR-18a, we generated miR-18a-knockout $\mathrm{SiHa}$ cell lines using the CRISPR/Cas9 gene editing system by transfecting the lentiCRISPR/Cas9-miR-18a vectors containing the individual sgRNAs into SiHa cells (Supplementary Fig. S4c, d). The T7EN1 assay [25] showed the cleavage bands, suggesting that CRISPR/Cas9 induced mutations at the $m i R-18 a$ locus (Supplementary Fig. S4e). Among the miR-18a-knockout clones, we identified two clones that carried a 4-bp deletion or a 10-bp deletion (Supplementary Fig. S4f). Deletion of 4 nucleotides significantly reduced and deletion of 10 nucleotides dramatically reduced (by more than 90\%) the expression of mature miR-18a in SiHa cells (Supplementary Fig. S4g). miR-18a knockout significantly repressed CC cell proliferation and invasion (Supplementary Fig. S4h, i).
To determine the effects of PD-L1 disruption in vivo, nude mice were subcutaneously injected with $\mathrm{SiHa}$ cells with PDL1-knockout or control cells. Mice injected with PD-L1knockout cells developed smaller subcutaneous tumors than those injected with control cells (Supplementary Fig. S4j, $\mathrm{k}$ ), indicating that miR-18a promotes a metastatic phenotype in CC cells.

\section{miR-18a enhances PD-L1 expression by repressing PTEN and WNK2}

By combining target prediction and qRT-PCR profiling, we found gene transcripts that were downregulated by miR-18a overexpression in CaSki cells and that were upregulated by miR-18a knockdown in $\mathrm{PaCa}-2$ cells, and identified five 
a

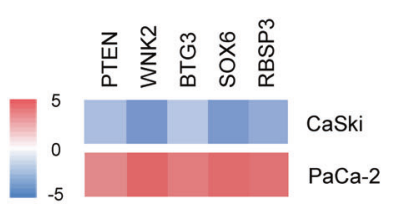

b
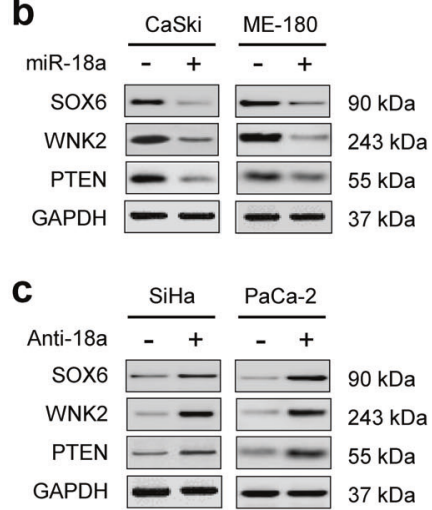

h

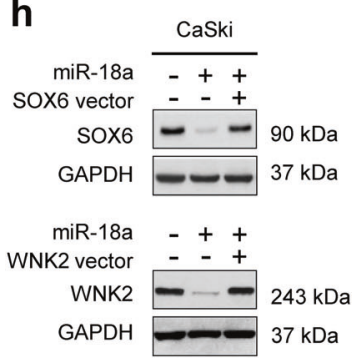

d

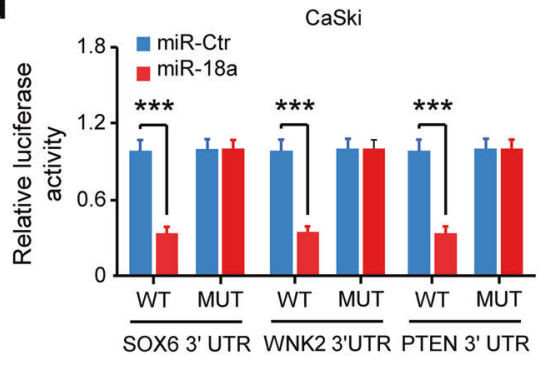

f

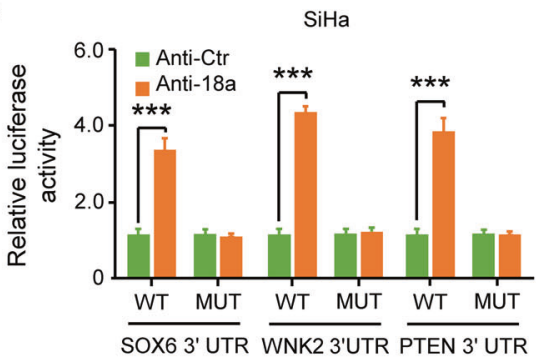

e

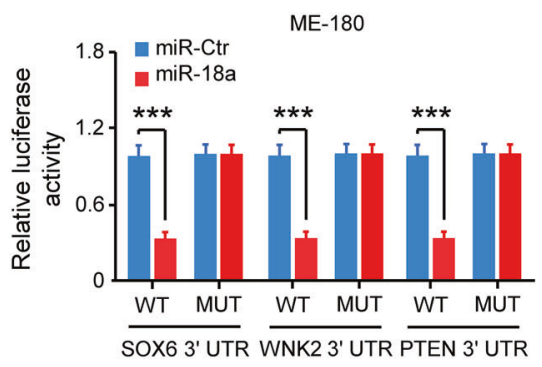

9

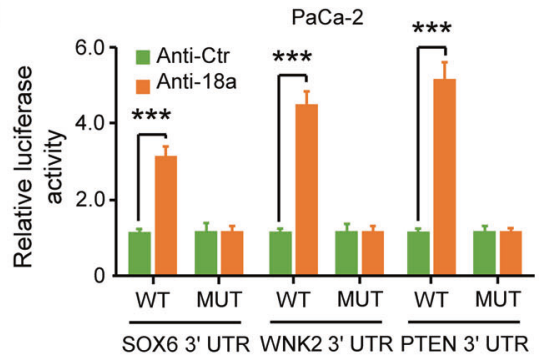

i

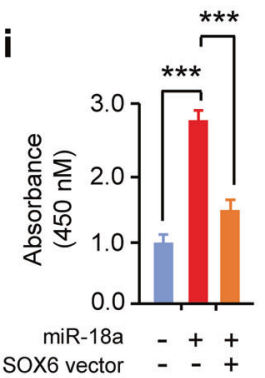

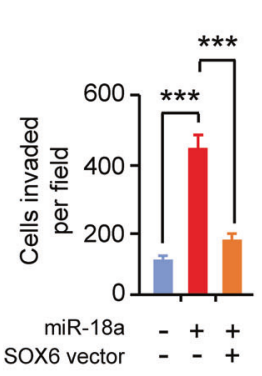

j

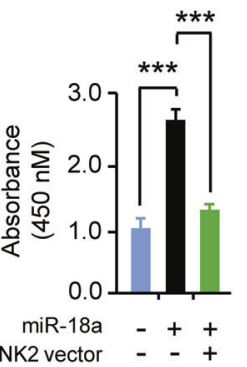

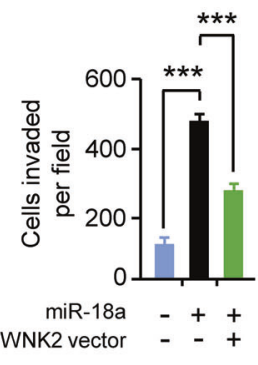

CaSki

Fig. 3 miR-18a directly targets the PTEN, SOX6, and WNK2 tumor suppressors. a Heat map of qRT-PCR data showing the expression of five genes (PTEN, WNK2, BTG3, SOX6, and RBSP3) that were downregulated by miR-18a overexpression in CaSki cells, but were upregulated by miR-18a knockdown in PaCa- 2 cells $(n=3)$. Red: upregulation of mRNA; green: downregulation of mRNA. b, $\mathbf{c}$ Western blotting analysis of SOX6, WNK2, and PTEN in CaSki and ME180 cells transfected with the miR-18a mimic or its negative control (b) and in $\mathrm{SiHa}$ and $\mathrm{PaCa}-2$ cells transfected with the anti-miR-18a inhibitor or its negative control $(\mathbf{c})(n=3)$. d-g Luciferase reporter

candidate genes (PTEN, WNK2, SOX6, BTG3, and RBSP3) that we defined as candidate targets of miR-18a (Fig. 3a; Supplementary Figs. S1d, S5).

Since PTEN, WNK2, SOX6, BTG3, and RBSP3 have putative miR-18a-binding sites in their $3^{\prime}$-UTRs (Supplementary Fig. S6a) and act as tumor suppressors in CC [18, 26-28], we hypothesized that miR-18a enhances PD-L1 expression via targeting these candidate targets.

PTEN, SOX6, WNK2, BTG3, and RBSP3 protein expression was decreased in miR-18a-overexpressing CC cells but was increased in miR-18a-silenced cancer cells (Fig. 3b,c; Supplementary Fig. S6b). Luciferase reporter assays showed that reporter activities driven by the $3^{\prime}$-UTRs of PTEN, SOX6, WNK2, BTG3, and RBSP3 were attenuated assays of CaSki (d), ME-180 (e), SiHa (f), and PaCa-2 (g) cells cotransfected with wild-type (WT) or mutant (MU) SOX6, WNK2, and PTEN 3'-UTRs and the miR-18a mimic, anti-miR-18a inhibitor or the appropriate negative control as indicated $(n=4)$. h Western blotting analysis of SOX6 and WNK2 in CaSki cells co-transfected with the miR-18a mimic or its negative control and the SOX6, WNK2 expression vector or control $(n=3)$. $\mathbf{i}, \mathbf{j}$ Cell proliferation assays (i) and Matrigel invasion assays $(\mathbf{j})$ of CaSki cells transfected with the miR-18a mimic or its negative control with or without the SOX6 or WNK2 expression vector $(n=6)$. $* * * P<0.001$

by miR-18a overexpression but elevated by miR-18a silencing. Mutations of the $3^{\prime}$-UTRs of these genes abrogated the effects of miR-18a, suggesting that they are direct targets of miR-18a (Fig. 3d-g; Supplementary Fig. S6c, d). Moreover, individual ectopic expression of SOX6, WNK2, BTG3, and RBSP3 significantly inhibited miR-18a-induced $\mathrm{CC}$ cell proliferation and invasion (Fig. 3h-j; Supplementary Figs. S6e-h, S7a-c), indicating that PTEN, WNK2, SOX6, BTG3, and RBSP3 are functional and direct targets of miR-18a in CC cells.

Consistent with the inhibitory effect of PTEN on PD-L1 expression [12], western blotting analysis showed upregulation of PTEN and downregulation of p-AKT and PD-L1 in SiHa cells with CRISPR/Cas9-mediated depletion of 
miR-18a (Supplementary Fig. S4g), supporting the notion that miR-18a silencing activates the PI3K/AKT pathway by targeting PTEN, and thereby upregulating PD-L1 expression.

WNK2 negatively regulates the MEK/ERK pathway in $\mathrm{CC}$ cells [26]. We explored the role of the WNK2/MEK/ ERK pathway in regulating miR-18a-induced PD-L1 expression. Overexpression of miR-18a increased ERK1/2 phosphorylation and upregulated PD-L1 expression; however, forced WNK2 expression blocked miR-18a-induced MEK/ERK pathway activation and reduced PD-L1 expression (Supplementary Fig. S7d), suggesting that miR-18a induces PD-L1 levels by targeting WNK2 to activate the MEK/ERK pathway. To further support these findings, we treated CC cells with the MEK inhibitor PD0325901. Inhibition of the MEK/ERK pathway with PD0325901 resulted in decreased PD-L1 expression and attenuated miR-18a-enhanced CC cell proliferation and invasion (Supplementary Fig. S7e-g). These data suggest that miR-18a-mediated PTEN and WNK2 inhibition and subsequent activation of the PI3K/AKT and MEK/ERK pathways contributes to PD-L1 upregulation in CC cells.

\section{miR-18a increases PD-L1 levels by targeting SOX6 to activate the Wnt/ $\beta$-catenin pathway and inactivate p53 signaling}

SOX6 is a known inhibitor of the $\mathrm{Wnt} / \beta$-catenin pathway [26]. Thus, we speculated that miR-18a increases PD-L1 levels by modulating the SOX6/Wnt/ $\beta$-catenin pathway. miR-18a overexpression induced the expression of PD-L1, $\beta$-catenin, and STAT3, a downstream effector of the Wnt/ $\beta$ catenin pathway $[15,16]$. Such changes were reversed by treatment with XAV939, a Wnt/ $\beta$-catenin inhibitor (Supplementary Fig. S8a). XAV939 treatment decreased miR18a-induced CC cell proliferation and invasion (Supplementary Fig. S8b, c). Forced STAT3 expression upregulated PD-L1 levels in CC cells (Supplementary Fig. S8d). qRT-PCR analysis indicated that miR-18a overexpression led to changes in the expression of $\mathrm{Wnt} / \beta$-catenin pathwayrelated genes, including the downregulation of AXIN2 and the upregulation of cyclin D1 and WISP3 (Fig. 4a), suggesting that miR-18a regulates PD-L1 in CC through activation of $\mathrm{Wnt} / \beta$-catenin/STAT3 signaling.

We next determined whether SOX6 downregulates PDL1 expression by repressing $\mathrm{Wnt} / \beta$-catenin pathway activity. Western blotting analysis and TOP/FOP-Flash reporter assays revealed that overexpressing SOX6 inhibited the induction of active $\beta$-catenin levels and $\mathrm{Wnt} / \beta$-catenin activity by miR-18a and downregulated cyclin D1 and PDL1 induced by miR-18a overexpression in CC cells (Fig. 4b-d). Co-immunoprecipitation assay [29] showed that $\beta$ catenin coimmunoprecipitated with SOX6 in nuclear extracts from CC cells (Fig. 4e), demonstrating that SOX6 represses Wnt signaling and PD-L1 expression by binding to $\beta$-catenin.

Another possible mechanism by which SOX6 may antagonize Wnt// -catenin activity is by repressing WISP3, an activator of this pathway [30]. Transient overexpression of SOX6 reduced endogenous WISP3 levels in CC cells (Fig. 4f, g). We identified the SOX6-binding motif within the WISP3 promoter (Fig. 4h). Chromatin immunoprecipitation (ChIP)-qPCR assays confirmed chromatin occupancy of SOX6 at the promoter regions of WISP3 and c-Myc [27] (Fig. 4i). We co-expressed a SOX6 expression vector with the WT WISP3 promoter or a version harboring a mutation at the predicted SOX6 binding site in CC cells. Forced SOX6 expression suppressed the activity of the WT, but not the mutant, WISP3 promoter compared with the controls (Fig. 4j). The inhibition of $\mathrm{Wnt} / \beta$-catenin activity, PD-L1 expression, and $\mathrm{CC}$ cell proliferation and invasion by SOX6 were reversed by ectopic WISP3 expression (Fig. 4k; Supplementary Fig. S9), suggesting that SOX6 increases PD-L1 expression by inhibiting WISP3.

SOX6 is considered an activator of the p53 pathway [27], and p53 suppresses PD-L1 expression via miR-34a [22]. We questioned whether SOX6 regulates PD-L1 expression by elevating p53 and miR-34 levels. Overexpression of SOX6 increased the levels of p53, p21, and miR-34a but decreased PD-L1 expression. These changes were partially reversed by p53 knockdown in CC cells (Fig. 4l, m). Introduction of miR-34a reduced PD-L1 expression and CC cell proliferation and invasion (Fig. 4n; Supplementary Fig. S10). These data reveal that miR-18a increases PD-L1 levels by targeting SOX6 to activate the $\mathrm{Wnt} / \beta$-catenin pathway and inactivate p53/miR-34a signaling.

\section{OCT4 transactivates miR-18a in CC cells}

Using the TRANSFAC database, we detected the binding sites for the transcriptional factors such as OCT4 and E2F1 [31], and found a positive correlation between OCT4 and miR-18a expression in CC cell lines (Supplementary Fig. S11a, b), indicating that OCT4 might positively regulate miR-18a expression. Transient overexpression of OCT4 increased miR-18a and PD-L1 levels, and siRNA-mediated silencing of OCT4 reduced miR-18a and PD-L1 expression (Supplementary Fig. S11c, d). ChIP-qPCR assays showed the binding of OCT4 to the promoter regions of $m i R-18 a$ and miR-125b [32] in CC cells (Supplementary Fig. S11e). The luciferase reporter assays revealed that the luciferase activity of the WT, but not the mutant, miR-18a promoter was increased by ectopic OCT4 expression but decreased by OCT4 depletion (Supplementary Fig. S11f). Overexpression of OCT4 increased CC cell proliferation and invasion, but inhibition of miR-18a with an anti-miRNA 
a

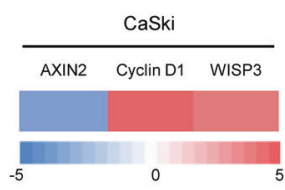

f

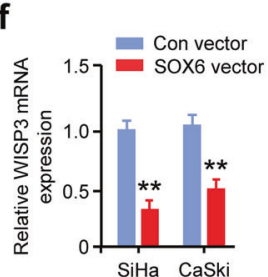

j

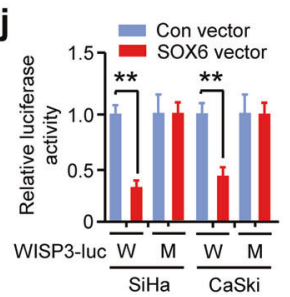

b

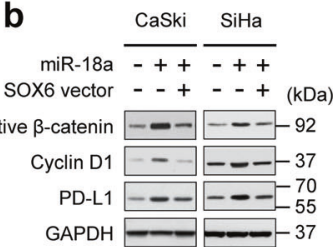

g
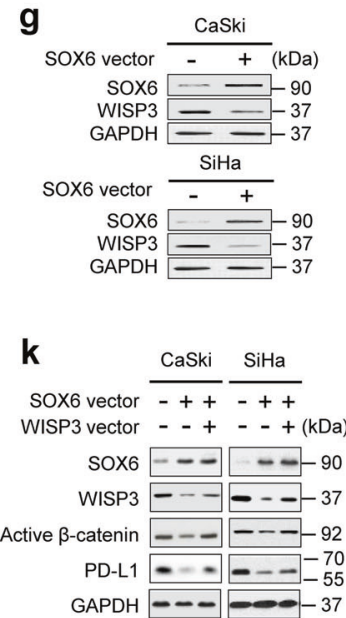

C

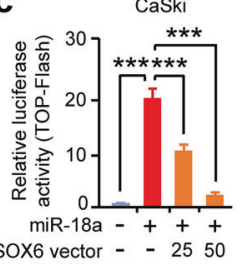

h

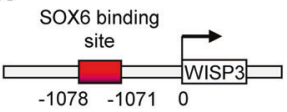

CTCAGGCCTAAGCGAGGCTTGAT GTTGTCTITATACAGTAGTGACTG CCTTGAACAGTAACAAAGGTGA GCAATCGGTATAGGATAGGTAAG GCAGCAGGAAAAGGAGGGATA

\section{|}

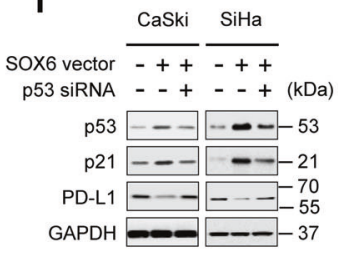

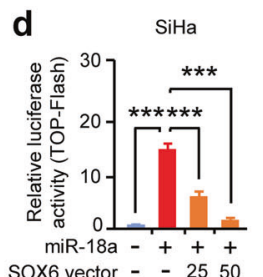

Sox6 vector - - 2550

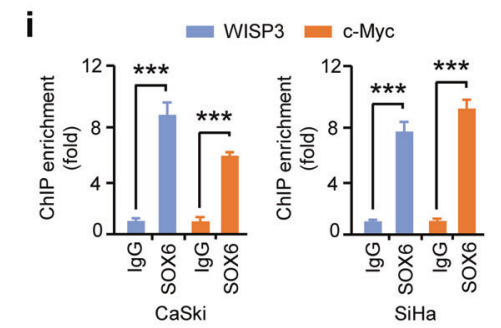

m

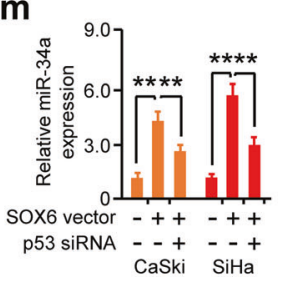

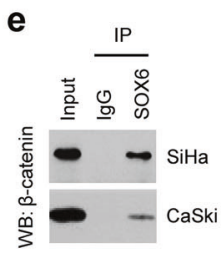

n

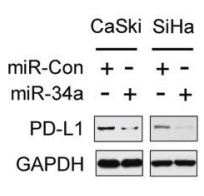

Fig. 4 miR-18a increases PD-L1 levels by repressing SOX6 to activate the $W n t / \beta$-catenin pathway and inactivate $p 53$ signaling. a Heat map of qRT-PCR data showing the expression of Wnt signaling pathwayrelated genes in CaSki cells transfected with the miR-18a mimic or its negative control. Red: upregulation of mRNA; green: downregulation of mRNA. b Western blotting analysis of active $\beta$-catenin, cyclin D1, and PD-L1 in CaSki and SiHa cells transfected with the miR-18a mimic or its negative control with or without the SOX6 expression vector $(n=3)$. c, d TOP/FOP-Flash reporter assays of CaSki (c) and $\mathrm{SiHa}(\mathbf{d})$ cells co-transfected with the miR-18a mimic or its negative control and the SOX6 expression vector or control $(n=4)$. e Coimmunoprecipitation assays reveal associations between endogenous SOX6 and $\beta$-catenin in CC cells. Cell lysates are labeled as input. IgG was used as the control $(n=3)$. f, $\mathbf{g}$ qRT-PCR (f) and western blotting (g) of WISP3 expression in CaSki and SiHa cells transfected with the SOX6 expression vector or control vector $(n=3)$. h Schematic representation of the human WISP3 gene promoter. The predicted SOX6 binding site is shown in red, and the primer sequences for ChIP-

inhibitor blocked these OCT4-mediated effects (Supplementary Fig. S11g). Knockdown of OCT4 inhibited CC proliferation and invasion, and these suppressive effects were partially rescued by restoring miR-18a levels (Supplementary Fig. S11h). Thus, OCT4 transactivates and upregulates PD-L1 expression, thereby promoting CC cell proliferation and invasion.

\section{Prognostic impacts of PD-L1, OCT4, WNK2, SOX6, BTG3, and RBSP3 in CC}

By analyzing web-based microarray database (GENT, MethHC, and ONCOMINE) that provide gene or miRNA expression patterns across normal and CC tissues, we found that $O C T 4, \mathrm{miR}-18 \mathrm{a}$, and $P D-L 1$ expression levels were
qPCR assays are underlined. i SOX6 occupancy at its potential binding site in the WISP3 gene promoter was analyzed using ChIP-qPCR assays. c-Myc, a known SOX6 target, was used as a positive control $(n=3)$. j CaSki and SiHa cells were transfected with the wild-type (WT) or mutant (MU) WISP3-promoter luciferase plasmid with or without the SOX6 expression vector. Relative luciferase activity was assessed $(n=4)$. $\mathbf{k}$ Western blot analysis of SOX6, WISP3, active $\beta$ catenin, and PD-L1 in CaSki and SiHa cells transfected with SOX6 expression vector or control vector and WISP3 expression vector or the appropriate control $(n=3)$. I Western blot analysis of $\mathrm{p} 53, \mathrm{p} 21$, and PD-L1 in CaSki and $\mathrm{SiHa}$ cells transfected with the SOX6 expression vector or control vector in the presence or absence of p53 siRNA $(n=3)$. $\mathbf{m}$ qRT-PCR analysis of miR-34a expression in CaSki and SiHa cells transfected with the SOX6 expression vector in the presence or absence of $\mathrm{p} 53$ siRNA $(n=3)$. $\mathbf{n}$ Western blot analysis of PD-L1 in CaSki and SiHa cells transfected with the miR-34a mimic or its negative control $(n=3)$. $* * P<0.01, * * * P<0.001$

significantly upregulated, whereas $W N K 2$, SOX6, BTG3, and $R B S P 3$ levels were significantly downregulated in CC tissues compared with normal cervical tissues (Supplementary Fig. S12a). Moreover, we evaluated the prognostic impacts of these genes on patient outcomes. Sixty CC patients were classified into two groups using the median expression value as the cutoff. Patients with higher levels of $P D-L 1$ and OCT4 or lower levels of WNK2, SOX6, BTG3, and RBSP3 had much shorter overall survival (Supplementary Fig. S12b). Lastly, we investigated whether elevated miR-18a expression is associated with OCT4 upregulation and with PTEN, WNK2, SOX6, BTG3, and RBSP3 downregulation. qRT-PCR analysis revealed a positive correlation between the expression of miR-18a and OCT4, and an inverse correlation of between miR-18a and 
Fig. 5 Proposed model for PDL1 induction in CC. Loss of $\operatorname{miR}-140 / 142 / 340 / 383$ contributes to PD-L1 upregulation in CC. Once miR18 a expression is triggered by OCT4 overexpression, miR-18a enhances PD-L1 levels by targeting PTEN, WNK2 (ERK1/ 2 pathway inhibitor), and SOX6 (Wnt/ $\beta$-catenin pathway inhibitor and p53 pathway activator) to activate the $\mathrm{PI} 3 \mathrm{~K} /$ AKT, MEK/ERK, and $\mathrm{Wnt} / \beta$ catenin pathways and inhibit the p53 pathway, therefore promoting tumor growth and invasion

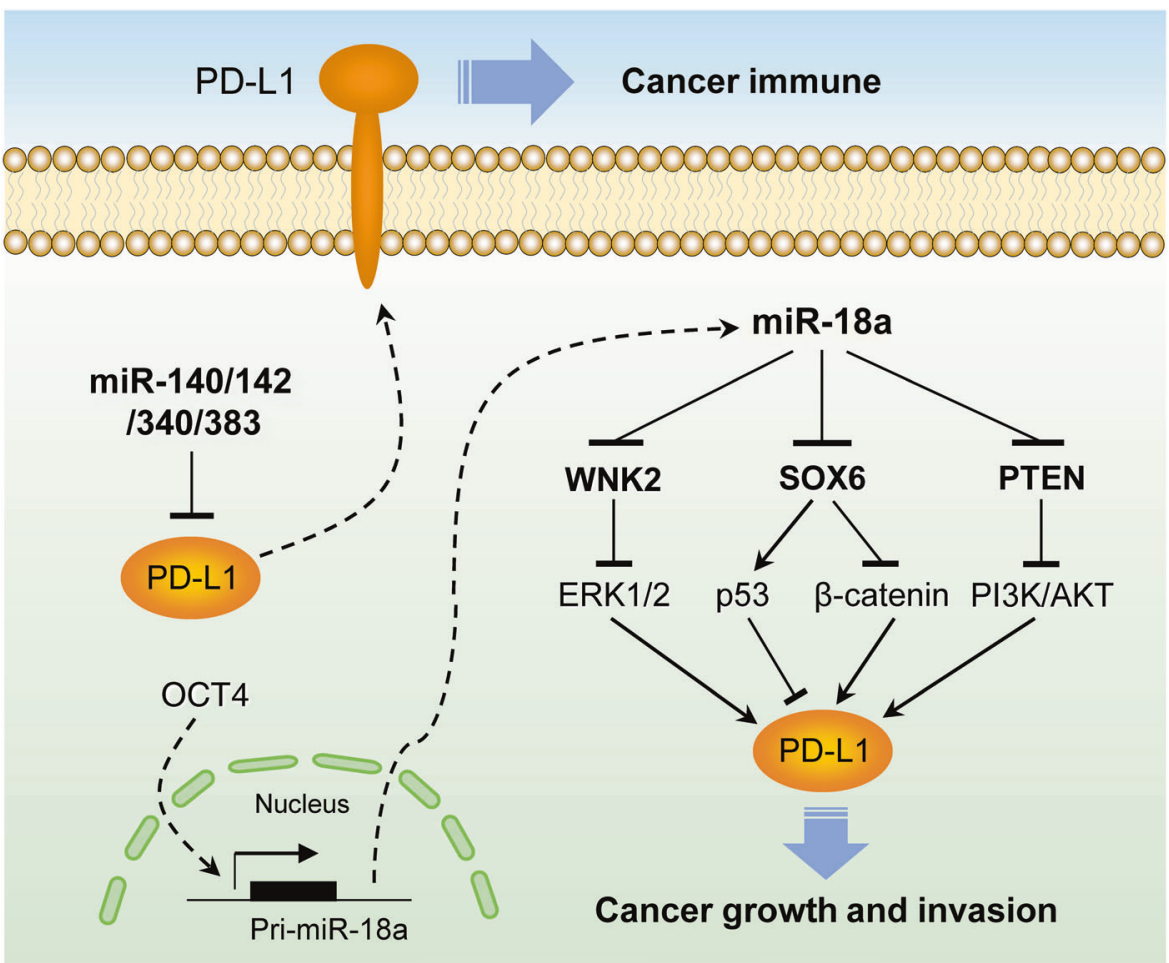

PTEN, WNK2, SOX6, BTG3, or RBSP3 levels in CC samples (Supplementary Fig. S13c). Altogether, these data support the utilization of PD-L1, OCT4, WNK2, SOX6, BTG3, and RBSP3 levels in clinical outcome predictions of CC.

\section{Discussion}

The present study shows that PD-L1 functions as a powerful cancer driver by stimulating cancer proliferation and invasion in vitro and facilitating cancer growth in vivo, providing a new insight into the involvement of PD-L1 in CC metastasis. Our results highlighted the crucial roles of miR140/142/340/383 downregulation and miR-18a upregulation in elevating PD-L1 expression and promoting CC progression. OCT4 induces the transcription of miR-18a, which targets multiple tumor suppressors, enhances PI3K/ AKT, MEK/ERK, and Wnt/ $\beta$-catenin pathway activity, and attenuates p53 signaling, finally leading to PD-L1 upregulation (Fig. 5).

MiRNAs were shown to target PD-L1 [20-22]. We demonstrated that miR-140/142/340/383 are direct suppressors of PD-L1. miR-140/142 were shown to function as tumor suppressors in CC [33, 34]. We also identified two previously uncharacterized miRNAs (miR-340/383) that inhibit $\mathrm{CC}$ cell proliferation and invasion by targeting PD-L1.
The PI3K/AKT, MAPK, and Wnt/ $\beta$-catenin pathways trigger the nuclear translocation of various transcription factors, and binding of the transcription factors such as STAT3 to the $P D-L 1$ promoter induces the transcription and translation of PD-L1 [11]. We demonstrate that OCT4activated miR-18a indirectly upregulates PD-L1 by targeting PTEN, WNK2, and SOX6 to activate the PI3K/AKT, MEK/ERK, and Wnt/ $\beta$-catenin pathways and repress p53 signaling. Consistently, dysregulation of PI3K/AKT pathway, MEK/ERK pathway, and p53 pathway represents critical mechanisms of tumor immune escape [35-37].

miR-18a, a member of the miR-17-92 cluster, is frequently overexpressed in human cancers and associated with cancer progression [38]. A previous study reported miR-18a upregulation in CC samples compared with normal cervical samples [39]. We showed here that miR-18a stimulates the metastatic ability of CC cells, and verified therapeutic effectiveness of CRISPR/Cas9-mediated miR18a disruption in a xenograft mouse model of $\mathrm{CC}$, suggesting that miR-18a-targeted therapy is a potential approach for treating patients with CC. The low miR-18a expression in normal cervical tissues may indicate that miR18a-targeted therapy will be characterized by high specificity and limited toxicity.

Several mechanisms have been shown to sustain miR18a expression in cancer cells. Activation of the transcription factors STAT3 and E2F1 induces miR-18a expression through direct binding [30, 40], highlighting the fact that 
our findings on OCT4 do not represent the only mechanism responsible for miR-18a overexpression in $\mathrm{CC}$. Interestingly, BTG3, a miR-18a target gene identified in this study, can directly bind and inhibit E2F1 [41], indicating the presence of a miR-18a-BTG3-E2F1 feedback loop that leads to persistent miR-18a overexpression in CC cells.

This study uncovers two previously unappreciated mechanisms contributing to PD-L1 overexpression in CC, the miR-140/142/340/383-PD-L1 axis, and the OCT4-miR18a-PTEN/WNK2/SOX6 oncogenic axis, thereby providing insights into epigenetic/genetic mechanisms underlying the pathogenesis of $\mathrm{CC}$, and highlighting the clinical potential of miRNA-based CC therapy.

\section{Materials and methods}

\section{Cell culture, reagents, and transient transfection}

The CC cell lines (CaSki, ME-180, and SiHa) and pancreatic cancer cell lines (AsPC-1 and $\mathrm{PaCa}-2$ ) were purchased from the ATCC. The immortalized human endometrial epithelial EM cell line was kindly provided by Satoru Kyo (Shimane University, Japan). Cells were cultured under the recommended conditions. Highly invasive SiHa cells were selected using Matrigel invasion chambers as previously described [24]. These cell lines were routinely tested by PCR for mycoplasma contamination. The MEK inhibitor PD0325901 and the Wnt/beta-catenin inhibitor XAV939 were obtained from Sigma-Aldrich. CC cells were treated with PD0325901 $(1 \mu \mathrm{M})$ for $48 \mathrm{~h}$ or with XAV939 ( 4 or $8 \mu \mathrm{M}$ ) for $16 \mathrm{~h}$. The miRNA mimics, anti-miRNA inhibitors, siRNAs against PD-L1, p53, and OCT4 and negative control siRNA were obtained from Ambion (Austin, TX). Transient transfection was accomplished with using Lipofectamine 3000 (Invitrogen, Carlsbad, CA) according to the manufacturer's instructions. The cDNA vectors encoding human PD-L1, SOX6, WNK2, BTG3, RBSP3, WISP3, or OCT4 were from OriGene (Rockville, MD). To generate stable cell lines overexpressing PD-L1, CaSki cells were transfected with a human PD-L1 cDNA expression vector (Origene) using Lipofectamine 3000 (Invitrogen). The respective control vector was used to generate control cell lines. After $24 \mathrm{~h}$, stable transfectants were selected in medium containing $0.5 \mathrm{mg} / \mathrm{ml}$ G418 (Sigma-Aldrich). The lentiviral vector harboring shRNA against human PD-L1 and the appropriate control were purchased from the shRNA/ORF Core Facility (MD Anderson Cancer Center). SiHa cells with stable PD-L1 knockdown were generated as previously reported [42]. The expression vectors harboring either full-length or cytoplasmic domain-truncated $P D-L 1$ gene were constructed and stable cell lines were established as previously reported [43].

\section{CC specimens and IHC staining}

All work related to human tissues was performed under protocols approved by the Institutional Review Board at Sun Yat-Sen University Cancer Center, China. Informed consent was obtained from all patients. Twenty-three paired human CC tissues and adjacent non-tumor tissues were collected at Sun Yat-Sen University Cancer Center. IHC staining was performed using antibodies against PD-L1 (clone E1L3N, Cell Signaling) as previously described [44]. Non-specific IgG was used as a negative control. A semiquantitative staining score ranging from 0 to 8 was calculated for each specimen by combining the staining intensity (0: negative; 1: low; 2 : moderate; and 3 : high) and the percentage of positive cells $(0$ : negative; $1+: 0-1 \% ; 2+$ : 1-10\%; 3+: 11-33\%; 4+: 34-66\%; and 5+: 67-100\%). Further, the degree of PD-L1 expression was quantified as follows: Negative expression (0 score), weak expression (1-3 scores), or strong expression (4-8 scores). In addition, a total of 60 pairs of snap-frozen primary CC specimens and adjacent non-tumor cervical tissues were processed for qRT-PCR analysis. Written informed consent was obtained from all patients.

\section{qRT-PCR analysis of miRNA and mRNAs}

Total RNA was isolated using TRIzol (Invitrogen). qRTPCR analysis of miRNA or mRNA was performed as previously reporetd $[45,46]$.

\section{Western blotting}

Cell protein extracts were prepared using M-Per Mammalian Protein Extraction Reagent (Pierce, Rockford, IL) according to the manufacturer's instructions. Total protein was fractionated by SDS-PAGE and transferred onto PVDF membranes. The following primary antibodies were used: PD-L1 (17952-1-AP, ProteinTech), SOX6 (NBP1-85811, Novus), WNK2 (07-2261, Millipore), BTG3 (ab112938, Abcam), RBSP3 (ab106973, Abcam), active $\beta$-catenin (05665, Millipore), OCT4 (ab19857, Abcam). p-AKT (sc293125), p-ERK1/2 (sc-16982), total $\beta$-catenin (sc-7963), ERK1/2 (sc-514302), PTEN (sc-7974), cyclin D1 (sc8396), p21 (sc-6246), and GAPDH (sc-47724) were obtained from Santa Cruz Biotechnology. GAPDH was analyzed to show equal protein loading. Blots were developed with the enhanced chemiluminescence blotting analysis system (Amersham Pharmacia Biotech, Buckinghamshire, UK). Immunoblot images were digitized 
and quantified using the ImageJ software. Results were expressed as a relative ratio of PD-L1 to GAPDH and the PD-L1/GAPDH ratio in normal cells was set as 1 .

\section{Cell proliferation assay, colony formation assay, and Matrigel invasion assay}

Cell proliferation was determined using the Cell Counting Kit-8 (Dojindo, Kumamoto, Japan) according to the manufacturer's protocol. In the colony formation assay, 500 cells were added to each well of a 6-well culture plate. After 14 days of culture, cells were fixed using $10 \%$ formalin and then stained using 10\% Giemsa. Colony formation efficiency was calculated as follows: Plate colony formation efficiency $(\%)=$ (number of colonies/number of cells inoculated) $\times 100$. Cell invasion was monitored and analyzed as described previously $[45,46]$.

\section{Tumor xenograft experiments}

All experiments involving mice were performed in accordance with the guidelines of the Animal Care and Use Committee of Sun Yat-Sen University Cancer Center. Fiveweek-old female nude mice (BALB/c) were purchased from Shanghai Laboratory Animal Center (Chinese Academy of Sciences, Shanghai, China). SiHa cells $\left(1 \times 10^{6}\right)$ were suspended in phosphate buffered saline $(100 \mu \mathrm{l})$ and then injected subcutaneously into mice. Tumor size was monitored using digital calipers, and tumor volume was calculated as length $\times$ width $^{2} \times 0.5$. All the mice were euthanized at day 35 post-inoculation. The excised tumors were embedded in paraffin and then subjected to IHC analysis of Ki-67 (ab15580, Abcam).

\section{Microarray experiment}

miRNA expression profiling was performed using Superprint G3 Human GE 8x60k Microarrays (Agilent Technologies) as previously described [44]. Microarray expression data were imported into GeneSpring software (Agilent Technologies). Differentially expressed miRNAs and mRNAs $(P<0.05$ and fold change $>2.0)$ were selected for further analysis. Microarray data was shown in Supplementary Table S1.

\section{Generation of PD-L1 knockout cells using the CRISPR/Cas9 system}

To knockout the endogenous miR-18a gene in SiHa cells, we used CRISPR/Cas9-mediated genome editing as previously described [47]. In brief, two gRNAs designed using CRISPR DESIGN (http://crispr.mit.edu/) to target the human miR-18a genomic sequence were subcloned into the lenti-CRISPR vector, and the inserted gRNAs were verified by DNA sequencing (gRNA-1: 5'-TATGCCAGAAGGAGCACTTA-3'; gRNA-2: 5'-TTATGCCAGAAGGAGCACTT- ${ }^{\prime}$ ). Stable cell lines were generated by transducing $\mathrm{SiHa}$ cells with the lenti-CRISPR-miR-18a vector and selecting transduced cells with $1 \mu \mathrm{g} \mathrm{ml}^{-1}$ puromycin (Sigma-Aldrich). SiHa cells transduced with a lentiCRISPR-control vector expressing a gRNA targeting GFP were used as the control.

\section{Luciferase activity assay}

The 3'-UTR of $P D-L 1$ was amplified from human genomic DNA and cloned downstream of the luciferase gene in the pMIR-reporter luciferase vector (Ambion, Austin, TX) as previously reported [48]. To construct the WISP3 or primiR-18a promoter luciferase vector, the promoter region encompassing the SOX6 or OCT4 binding site was amplified from human genomic DNA and cloned into the MluI/ BglII sites of the pGL3 vector (Promega). The potential miR-140/142/340/383 binding sites in the $3^{\prime}$-UTR of PDL1 and the potential SOX6 and OCT4 binding sites in the WISP3 and pri-miR-18a promoter regions were mutated using a QuickChange Site-Directed Mutagenesis Kit (Stratagene, La Jolla, CA). ImiRP was used for mutating miRNA binding sites that are located in the $3^{\prime}$-UTR of target genes [49]. Luciferase activity was measured $48 \mathrm{~h}$ after transfection using a Dual Luciferase Assay Kit (Promega).

\section{TOP-Flash/FOP-Flash reporter assay}

The TOP-Flash reporter and pTK-RL plasmids were cotransfected into CC cells $\left(5 \times 10^{4}\right)$ in 24 -well plates with the miR-18a mimic and a SOX6 expression vector, and the activity of the Firefly and Renilla luciferase reporters was determined at $48 \mathrm{~h}$ after transfection using a Dual Luciferase Assay Kit (Promega). TOP-Flash reporter activity is presented as the relative ratio of Firefly luciferase activity to Renilla luciferase activity.

\section{ChIP-qPCR assay}

Cellular chromatin was immunoprecipitated with an antibody against SOX6 (ab30455, Abcam), OCT4 (ab19857, Abcam), or IgG (control, Santa Cruz Biotechnology) using the Pierce Agarose ChIP Kit (Pierce). The amount of immunoprecipitated DNA was quantified using Takara SYBR Premix Ex Taq II (Takara). The results are presented as the fold enrichment over the $\operatorname{IgG}$ control. The human $c$ Myc [27] and miR-125b [32] promoters were used as positive controls for SOX6 and OCT4 binding, respectively. 


\section{Statistical analysis}

Statistical analyses were performed using SPSS version 22.0 (SPSS Inc., Chicago, IL, USA). All results are expressed as mean \pm s.d. All experiments were performed at least three times. Statistical differences between categorical data were evaluated by Fisher's exact test. Comparisons between groups of continuous variables were performed using Student's $t$-test ( 2 groups) or one-way ANOVA test ( $>2$ groups). The Wilcoxon signed-rank test was used to compare two groups of paired nonparametric data. Differences in mRNA or miRNA expression between CC tissues and normal cervical tissues were evaluated using the nonparametric Mann-Whitney U test. Results with two-tailed $P$-values $<0.05$ were judged to be statistically significant.

Acknowledgements This research was supported by a grant from the Department of Women's Health Educational System, JSPS Grant-in-Aid for Scientific Research (C) (15K10697 and 16K11123) and the Science and Technology Planning Project of Guangdong Province, China (2014A020212124). We thank Dr. Zhujie Xu for experimental assistance.

\section{Compliance with ethical standards}

Conflict of interest The authors declare that they have no conflict of interest.

Open Access This article is licensed under a Creative Commons Attribution 4.0 International License, which permits use, sharing, adaptation, distribution and reproduction in any medium or format, as long as you give appropriate credit to the original author(s) and the source, provide a link to the Creative Commons license, and indicate if changes were made. The images or other third party material in this article are included in the article's Creative Commons license, unless indicated otherwise in a credit line to the material. If material is not included in the article's Creative Commons license and your intended use is not permitted by statutory regulation or exceeds the permitted use, you will need to obtain permission directly from the copyright holder. To view a copy of this license, visit http://creativecommons. org/licenses/by/4.0/.

\section{References}

1. Siegel RL, Miller KD, Jemal A. Cancer statistics, 2015. CA Cancer J Clin. 2015;65:5-29.

2. Mehlen P, Puisieux A. Metastasis: a question of life or death. Nat Rev Cancer. 2006;6:449-58.

3. Chen L, Han X. Anti-PD-1/PD-L1 therapy of human cancer: past, present, and future. J Clin Invest. 2015;125:3384-91.

4. He J, Hu Y, Hu M, Li B. Development of PD-1/PD-L1 pathway in tumor immune microenvironment and treatment for non-small cell lung cancer. Sci Rep. 2015;5:13110.

5. Heong V, Ngoi N, Tan DS. Update on immune checkpoint inhibitors in gynecological cancers. J Gynecol Oncol. 2017;28:e20.

6. Wang X, Teng F, Kong L, Yu J. PD-L1 expression in human cancers and its association with clinical outcomes. Onco Targets Ther. 2016;9:5023-39.

7. Mezache L, Paniccia B, Nyinawabera A, Nuovo GJ. Enhanced expression of PD L1 in cervical intraepithelial neoplasia and cervical cancers. Mod Pathol. 2015;28:1594-602.
8. Song X, Liu J, Lu Y, Jin H, Huang D. Overexpression of B7-H1 correlates with malignant cell proliferation in pancreatic cancer. Oncol Rep. 2014;31:1191-8.

9. Abiko K, Matsumura N, Hamanishi J, Horikawa N, Murakami R, Yamaguchi $\mathrm{K}$, et al. IFN- $\gamma$ from lymphocytes induces PD-L1 expression and promotes progression of ovarian cancer. $\mathrm{Br} \mathbf{J}$ Cancer. 2015;112:1501-9.

10. Li J, Chen L, Xiong Y, Zheng X, Xie Q, Zhou Q, et al. Knockdown of PD-L1 in human gastric cancer cells inhibits tumor progression and improves the cytotoxic sensitivity to CIK therapy. Cell Physiol Biochem. 2017;41:907-20.

11. Wang Y, Wang H, Zhao Q, Xia Y, Hu X, Guo J. PD-L1 induces epithelial-to-mesenchymal transition via activating SREBP-1c in renal cell carcinoma. Med Oncol. 2015;32:212.

12. Chen J, Jiang CC, Jin L, Zhang XD. Regulation of PD-L1: a novel role of pro-survival signalling in cancer. Ann Oncol. 2016;27:409-16.

13. Sharma P, Hu-Lieskovan S, Wargo JA, Ribas A. Primary, adaptive, and acquired resistance to cancer immunotherapy. Cell. 2017;168:707-23.

14. Liang X, Fu C, Cui W, Ober-Blöbaum JL, Zahner SP, Shrikant $\mathrm{PA}$, et al. $\beta$-catenin mediates tumor-induced immunosuppression by inhibiting cross-priming of $\mathrm{CD}^{+} \mathrm{T}$ cells. J Leukoc Biol. 2014;95:179-90.

15. Yan S, Zhou C, Zhang W, Zhang G, Zhao X, Yang S, et al. Betacatenin/TCF pathway upregulates STAT3 expression in human esophageal squamous cell carcinoma. Cancer Lett. 2008;271:85-97.

16. Anand M, Lai R, Gelebart P. $\beta$-catenin is constitutively active and increases STAT3 expression/activation in anaplasticlymphoma kinase-positive anaplastic large cell lymphoma. Haematologica. 2011;96:253-61.

17. Ribeiro J, Sousa H. MicroRNAs as biomarkers of cervical cancer development: a literature review on miR-125b and miR-34a. Mol Biol Rep. 2014;41:1525-31.

18. Xiong Y, Sun F, Dong P, Watari H, Yue J, Yu MF, et al. iASPP induces EMT and cisplatin resistance in human cervical cancer through miR-20a-FBXL5/BTG3 signaling. J Exp Clin Cancer Res. 2017;36:48

19. Fujita Y, Yagishita S, Hagiwara K, Yoshioka Y, Kosaka N, Takeshita F, et al. The clinical relevance of the miR-197/CKS1B/ STAT3-mediated PD-L1 network in chemoresistant non-smallcell lung cancer. Mol Ther. 2015;23:717-27.

20. Gong AY, Zhou R, Hu G, Li X, Splinter PL, O'Hara SP, et al. MicroRNA-513 regulates B7-H1 translation and is involved in IFN-gamma-induced B7-H1 expression in cholangiocytes. J Immunol. 2009;182:1325-33.

21. Guo W, Tan W, Liu S, Huang X, Lin J, Liang R, et al. MiR-570 inhibited the cell proliferation and invasion through directly targeting B7-H1 in hepatocellular carcinoma. Tumour Biol. 2015;36:9049-57.

22. Cortez MA, Ivan C, Valdecanas D, Wang X, Peltier HJ, Ye Y, et al. PDL1 regulation by $\mathrm{p} 53$ via miR-34. J Natl Cancer Inst. 2015;108:djv303.

23. Deng J, Zhang W, Liu S, An H, Tan L, Ma L. LATS1 suppresses proliferation and invasion of cervical cancer. Mol Med Rep. 2017;15:1654-60.

24. Dong P, Ihira K, Xiong Y, Watari H, Hanley SJ, Yamada T, et al. Reactivation of epigenetically silenced miR-124 reverses the epithelial-to-mesenchymal transition and inhibits invasion in endometrial cancer cells via the direct repression of IQGAP1 expression. Oncotarget. 2016;7:20260-70.

25. Su S, Hu B, Shao J, Shen B, Du J, Du Y, et al. CRISPR-Cas9 mediated efficient PD-1 disruption on human primary T cells from cancer patients. Sci Rep. 2016;6:20070. 
26. Moniz S, Veríssimo F, Matos P, Brazão R, Silva E, Kotelevets L, et al. Protein kinase WNK2 inhibits cell proliferation by negatively modulating the activation of MEK1/ERK1/2. Oncogene. 2007;26:6071-81.

27. Wang J, Ding S, Duan Z, Xie Q, Zhang T, Zhang X, et al. Role of p14ARF-HDM2-p53 axis in SOX6-mediated tumor suppression. Oncogene. 2016;35:1692-702.

28. Mitra S, Mazumder Indra D, Bhattacharya N, Singh RK, Basu PS, Mondal RK, et al. RBSP3 is frequently altered in premalignant cervical lesions: clinical and prognostic significance. Genes Chromosomes Cancer. 2010;49:155-70.

29. Kramer HB, Lai CF, Patel H, Periyasamy M, Lin ML, Feller SM, et al. LRH-1 drives colon cancer cell growth by repressing the expression of the CDKN1A gene in a p53-dependent manner. Nucleic Acids Res. 2016;44:582-94.

30. Fang F, Zhao WY, Li RK, Yang XM, Li J, Ao JP, et al. Silencing of WISP3 suppresses gastric cancer cell proliferation and metastasis and inhibits Wnt/ $\beta$-catenin signaling. Int J Clin Exp Pathol. 2014;7:6447-61.

31. Sylvestre Y, De Guire V, Querido E, Mukhopadhyay UK, Bourdeau V, Major F, et al. An E2F/miR-20a autoregulatory feedback loop. J Biol Chem. 2007;282:2135-43.

32. Zheng Z, Qu JQ, Yi HM, Ye X, Huang W, Xiao T, et al. MiR$125 \mathrm{~b}$ regulates proliferation and apoptosis of nasopharyngeal carcinoma by targeting A20/NF- $\mathrm{BB}$ signaling pathway. Cell Death Dis. 2017;8:e2855.

33. Su Y, Xiong J, Hu J, Wei X, Zhang X, Rao L. MicroRNA-140-5p targets insulin like growth factor 2 mRNA binding protein 1 (IGF2BP1) to suppress cervical cancer growth and metastasis. Oncotarget. 2016;7:68397-411.

34. Jiang D, Wang H, Li Z, Li Z, Chen X, Cai H. MiR-142 inhibits the development of cervical cancer by targeting HMGB1. Oncotarget. 2017;8:4001-7.

35. Noh KH, Kang TH, Kim JH, Pai SI, Lin KY, Hung CF, et al. Activation of Akt as a mechanism for tumor immune evasion. Mol Ther. 2009;17:439-47.

36. Sumimoto H, Imabayashi $F$, Iwata $T$, Kawakami Y. The BRAFMAPK signaling pathway is essential for cancer-immune evasion in human melanoma cells. J Exp Med. 2006;203:1651-6.

37. Muñoz-Fontela C, Mandinova A, Aaronson SA, Lee SW. Emerging roles of p53 and other tumour-suppressor genes in immune regulation. Nat Rev Immunol. 2016;16:741-50.
38. Olive V, Jiang I, He L. mir-17-92, a cluster of miRNAs in the midst of the cancer network. Int $\mathrm{J}$ Biochem Cell Biol. 2010;42:1348-54.

39. Wilting SM, Snijders PJ, Verlaat W, Jaspers A, van de Wiel MA, van Wieringen $\mathrm{WN}$, et al. Altered microRNA expression associated with chromosomal changes contributes to cervical carcinogenesis. Oncogene. 2013;32:106-16.

40. Cai Y, Chen H, Mo X, Tang Y, Xu X, Zhang A, et al. Toxoplasma gondii inhibits apoptosis via a novel STAT3-miR-17-92-Bim pathway in macrophages. Cell Signal. 2014;26:1204-12.

41. Ou YH, Chung PH, Hsu FF, Sun TP, Chang WY, Shieh SY. The candidate tumor suppressor BTG3 is a transcriptional target of p53 that inhibits E2F1. EMBO J. 2007;26:3968-80.

42. Li CW, Lim SO, Xia W, Lee HH, Chan LC, Kuo CW, et al. Glycosylation and stabilization of programmed death ligand-1 suppresses T-cell activity. Nat Commun. 2016;7:12632.

43. Chen L, Xiong Y, Li J, Zheng X, Zhou Q, Turner A, et al. PD-L1 expression promotes epithelial to mesenchymal transition in human esophageal cancer. Cell Physiol Biochem. 2017;42:2267-80.

44. Konno Y, Dong P, Xiong Y, Suzuki F, Lu J, Cai M, et al. MicroRNA-101 targets EZH2, MCL-1 and FOS to suppress proliferation, invasion and stem cell-like phenotype of aggressive endometrial cancer cells. Oncotarget. 2014;5:6049-62.

45. Ihira K, Dong P, Xiong Y, Watari H, Konno Y, Hanley SJ, et al. EZH2 inhibition suppresses endometrial cancer progression via miR-361/Twist axis. Oncotarget. 2017;8:13509-20.

46. Dong P, Xiong Y, Watari H, Hanley SJ, Konno Y, Ihira K, et al. MiR-137 and miR-34a directly target Snail and inhibit EMT, invasion and sphere-forming ability of ovarian cancer cells. J Exp Clin Cancer Res. 2016;35:132.

47. Huo W, Zhao G, Yin J, Ouyang X, Wang Y, Yang C, et al. Lentiviral CRISPR/Cas9 vector mediated miR-21 gene editing inhibits the epithelial to mesenchymal transition in ovarian cancer cells. J Cancer. 2017;8:57-64.

48. Xu S, Tao Z, Hai B, Liang H, Shi Y, Wang T, et al. miR-424(322) reverses chemoresistance via $\mathrm{T}$-cell immune response activation by blocking the PD-L1 immune checkpoint. Nat Commun. 2016;7:11406.

49. Ryan BC, Werner TS, Howard PL, Chow RL. ImiRP: a computational approach to microRNA target site mutation. BMC Bioinforma. 2016;17:190. 